\title{
COMMUNICATION
}

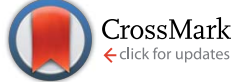

Cite this: RSC Adv., 2015, 5, 9462

Received 6th November 2014 Accepted 22nd December 2014

DOI: $10.1039 / c 4 r a 14001 j$

www.rsc.org/advances

\section{Structural lipid nanoparticles self-assembled from electrospun core-shell polymeric nanocomposites $\dagger$}

\author{
Deng-Guang $\mathrm{Yu}^{\text {*a }}{ }^{\mathrm{a}}$ Kenneth White, ${ }^{\mathrm{b}}$ Nicholas Chatterton, ${ }^{\mathrm{b}}$ Ying $\mathrm{Li}^{\mathrm{a}}{ }^{\mathrm{L}}$ Lingling $\mathrm{Li}^{\mathrm{a}}$ \\ and Xia Wang*a
}

\begin{abstract}
Electrospun polymeric core-shell nanocomposites are exploited as templates to manipulate molecular self-assembly for preparing structural lipid nanoparticles, during which the confinement effect of fibers together with their core-shell structure, the aqueous environment and the secondary interactions, all contributed synergistically to facilitate molecular self-aggregation to produce lipid nanoparticles with a drug entrapment efficiency of $95.9 \%$ with a sustained drug release profile.
\end{abstract}

Electrospun nanofibers have been demonstrated to be good templates for indirectly producing functional nano-objects such as inorganic nanotubes, carbon nanofibers and fibrous hydrogel materials with encapsulated microbes. ${ }^{1-6}$ These were realized through a strategy that takes the nanofibers as templates in a whole way by virtue of their physical configurations, i.e. through post-treatment of the nanofiber mats (for example by physical absorbance and removing the filament-forming polymer matrix, cross linking reactions, calcinations or carbonization).

Most recently, electrospun monolithic composite nanofibers were demonstrated to be good templates to directly manipulate the molecular self-assembly of multiple components for fabricating functional nano-objects in situ such as solid lipid nanoparticles and liposomes. ${ }^{7,8}$ In contrast to the aforementioned studies, these were achieved by the virtue of the nanometer confinement effect of the nanofibers and the formulation of a polymeric composite in which the functional building blocks can disperse throughout the polymer matrix on a molecular scale. Similarly, composite microparticles produced by electrospraying were also effective templates for producing self-

${ }^{a}$ School of Materials Science \& Engineering, University of Shanghai for Science and Technology, Shanghai 200093, P.R. China. E-mail:ydg017@usst.edu.cn; wangxia@ usst.edu.cn

${ }^{b}$ School of Human Sciences, Faculty of Life Sciences and Computing, London Metropolitan University, 166-220 Holloway Road, London N7 8DB, UK

$\dagger$ Electronic supplementary information (ESI) available: Experimental procedures, characterization methods, and Fig. S1-S5. See DOI: 10.1039/c4ra14001j assembled nanoparticles by virtue of their confinement effect on a microscale. ${ }^{9}$

Although all the nanofiber and composite microparticles have been demonstrated to be good templates in different ways and for different applications, they are products derived from single fluid electrohydrodynamic atomisation (EHDA - electrospraying, electrospinning and e-jet printing ${ }^{\mathbf{1 0}}$ ) processes, which lack secondary microstructure characteristics. Nano-particle self-assembly was achieved mainly through the properties of the components in the nanofibers (often their solubility in special solvents). One of the powerful capabilities of EHDA processes is that they can copy structures from the macro world to products at the micro/nano scale. ${ }^{\mathbf{1 1}}$ For example, through the interactions between the electrons and fluid liquid, electrospinning and electrospraying can easily duplicate the structure of macro jet devices (such as concentric, side-by-side and triaxial spinnerets) to generate products with special microstructures such as core-shell nanofibers/particles and side-by-side and tri-axial nanofibers. ${ }^{\mathbf{1 1 - 1 4}}$

Fibers and particles with secondary structure characteristics should be better templates for broader applications in manipulating molecular self-assembly, as well as designing and developing new advanced materials, than those generated by single fluid processes. Combining two or more fluid EHDA processes can overcome the difficulties of co-dissolving multiple components in a single solvent that often make a single fluid EHDA process a failure. Moreover, through manipulating the spatial distribution of building blocks in the structured fibers or particles, the molecular self-assembly processes may be controlled more accurately and it may result in self-assembled products with higher quality.

In this report, we describe the use of core-shell nanofibers produced from coaxial electrospinning as templates for molecular self-assembly to prepare drug-loaded core-shell nanoparticles in situ. The hydrophilic polymer polyvinylpyrrolidone K60 was used as the filament-forming matrix, and the lipophilic drug carrier tristearin (GTS) and a poorly 
water-soluble drug acyclovir (ACY) were used as functional building blocks to demonstrate the strategy.

The poorly water-soluble drug ACY also has poor solubility in a series of typical organic solvents, such as ethanol, methanol, chloroform and acetone, but it is soluble in $N, N^{\prime}$-dimethylacetamide (DMAc). ${ }^{15}$ PVP has no electrospinnablity in DMAc, thus it is impossible to prepare composite nanofibers of these multiple components using single fluid electrospinning owing to the lack of cosolubility of the components or good electrospinnability. For a traditional coaxial electrospinning process, the core solution does not need to have electrospinnability because the shell solution surrounds the core liquid and acts as a guide. ${ }^{16,17}$ Here, the shell solution is critical and the shell polymer-solvent system selected should be electrospinnable by itself to facilitate the formation of a core-shell structure within the fibers. Thus, although the core solution consisted of $10 \%$ $(\mathrm{w} / \mathrm{v})$ PVP and $2 \%(\mathrm{w} / \mathrm{v})$ ACY in a mixed solvent of DMAc : ethanol $(4: 6, v / v)$ and has no electrospinnability, the electrospinnable shell fluid, which consisted of $10 \%$ (w/v) PVP and $2 \%(\mathrm{w} / \mathrm{v})$ GTS in chloroform, can ensure a smooth coaxial electrospinning process and the formation of core-shell fibers with strategic and spatial deposition of building blocks in different parts of the fibers. More details about the co-axial electrospinning process can be found in the ESI. $\dagger$

The field emission scanning electron microscopy (FESEM) images of the fibers and their cross-section (Fig. 1a and b) demonstrate that the fibers have smooth surfaces and a homogeneous inner structure, with no particles separating out from the polymer matrix, either in the shell or in the core parts. Measurement of the fiber thickness using Image $J$ software indicated that the fibers have an average diameter of $960 \pm 130$ $\mathrm{nm}$. Transmission electron microscopy (TEM) images (Fig. 1c) demonstrate the obvious core-shell structure of the fibers, and the uniform gray shading of the shell, and the TEM images of core parts of the fibers suggest a homogeneous distribution of GTS and ACY in the PVP matrix in the different parts of the
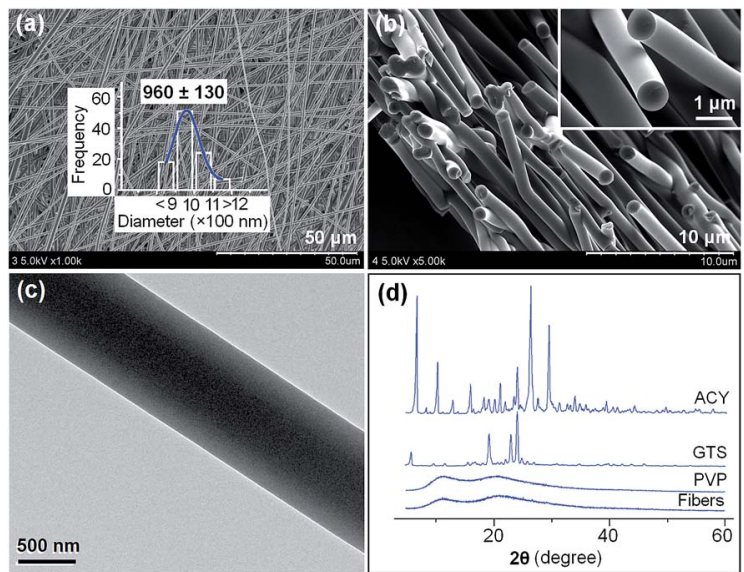

Fig. 1 Characterisation of the electrospun core-shell composite fibers: (a) FESEM image of the surface of the fiber; (b) FESEM images of the cross-section of fiber, the inset has a magnification of $\times 20000$; (c) TEM images of the core-shell structure of the fiber; (d) XRD patterns of ACY, GTS, PVP and fiber composites. fibers. Furthermore, X-ray diffraction (XRD) patterns (Fig. 1d) indicate that ACY and GTS had lost their original crystalline structures when incorporated into the fibers. The results from FESEM, TEM and XRD taken together demonstrate that the components in the composite core-shell fibers were highly mixed and had been converted to an amorphous state.

The distribution of building blocks in the filament-forming polymer matrix on a molecular scale is the first and foremost factor for the fibers to act as templates to direct molecular selfassembly. This ensures that the molecules can be transferred and they can spontaneously make contact in a micro-confined region when they are liberated in a suitable environment. For electrospun products, polymeric composites can be produced easily by directly exploiting electrical energy to dry and solidify fluid jets containing filament-forming polymer matrix and the guest active ingredient, which produces nano objects very rapidly, often in the order of $10^{-2} \mathrm{~s}^{18,19}$ Based on the favorable interactions between the components and the polymer matrix, the physical state of the components in the liquid solutions can be propagated into the solid nanofibers to form a composite.

To observe the self-assembly process, a drop of water was placed on fibers collected on a glass slide to initiate the molecular self-assembly process, and then it was allowed to dry in the air. Shown in Fig. 2a is an image observed using polarization microscopy under cross-polarized light, in which selfassembly events, "frozen" by drying, can be divided into three regions along the water extruding direction indicated by the white arrow. In region 3, there are only swelling fibers. In region 2 , there are many bright dots along the fiber lines that appear to be "cut" from the fibers. In region 1, there are many tiny bright dots randomly scattered on the slide. An FESEM image of region 1 is shown in Fig. 2b. The polymer matrix PVP formed some wrinkles and the self-assembled nanoparticles separated out from the fiber matrix and dispersed around it. The TEM images

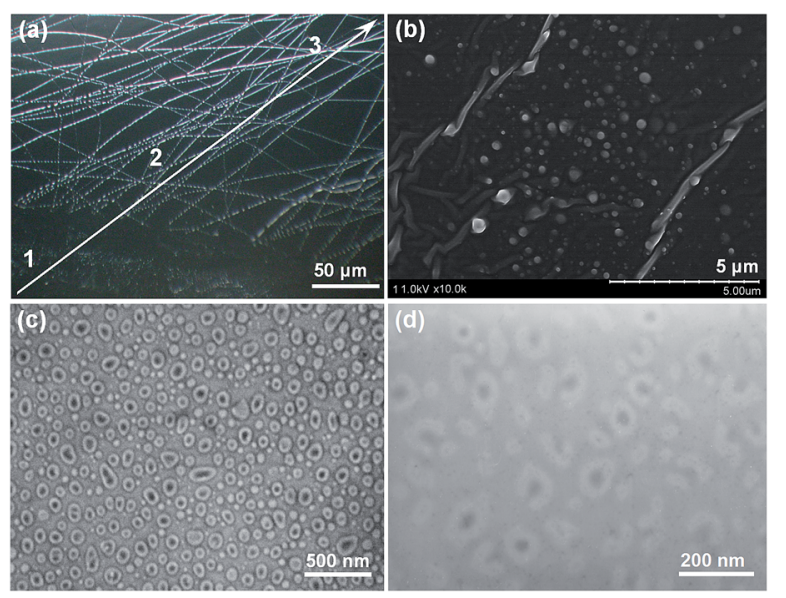

Fig. 2 Self-assembly and characterization of core-shell nanoparticles: (a) polarization microscopy observation of the self-assembly process occurring when a drop of water was placed on fibers collected on a glass slide (magnification of $7 \times 40$ ); the arrow shows the water extruding direction. (b) FESEM image of a naturally dried area of selfassembly. (c) and (d) TEM images of the nanoparticles at $80 \mathrm{kV}$ for $0.2 \mathrm{~s}$ with different magnifications. 
of core-shell nanoparticles in Fig. 2c and d demonstrate that the ACY was well encapsulated by GTS, following the template of the core-shell fibers, although there are some GTS nanoparticles without ACY, which are greatly smaller than the coreshell nanoparticles.

A static and dynamic light scattering analysis of the selfassembled lipid nanoparticles showed that they have an average diameter of $103 \pm 19 \mathrm{~nm}$ (Fig. 3a). During the selfassembly process, a small amount of ACY was also freed into the environmental water. The amount of free ACY in the supernatant from the suspensions was found to be $4.1 \% \pm$ $2.3 \%$, meaning that $95.9 \% \pm 2.3 \%$ of the drug was encapsulated into the structural nanoparticles (ESI $\dagger$ ). After $12 \mathrm{~h}$ of in vitro dissolution, $96.6 \%$ of the drug in the self-assembled nanoparticles was freed into the dissolution medium (Fig. 3b). According to the Peppas equation: ${ }^{20} Q=k t^{n}$, where $Q$ is the percentage of drug released at time $t, k$ is a kinetic constant and $n$ is the diffusional exponent indicative of the release mechanism. Drug release from the self-assembled lipid nanoparticles could be fitted with the equation $Q=33.11 t^{0.44}\left(R^{2}=0.9935\right)$. The value of the diffusion index $n$ was 0.44 , indicating that ACY release was mainly by a typical Fick diffusion mechanism.

In view of these observations, a self-assembly process can be proposed as follows (Fig. 4): (1) the self-assembly process begins with polymer swelling when PVP absorbs water, and the "anchored" building blocks are liberated from the polymerbased composites by water molecules; (2) as the hydrophilic fiber matrix further absorbs water and swells, the compact structure of fibers becomes looser; thus, the building blocks can randomly move in confined regions; (3) the hydrophobic building blocks spontaneously co-aggregate into hybrid "particles" locally due to repulsion forces from the surrounding aqueous environment, and most of the "flexible" PVP molecules that underwent disentanglement are also displaced in the "particles"; (4) the hydrophilic polymer molecules leave the "particle" and dissolve in the dissolution medium; moreover, the "particles" condense into nanoparticles. This is why the dots in region 2 are considerably bigger than those in region 1 , as shown in Fig. 2a. The sequence of events is that first "particles" are formed when the fibers break up, and then they form smaller "nanoparticles" through the removal of the polymer molecules by water. This suggests that the hydrophobic interactions between the building blocks and the water environment play the key role during the self-assembly process. This, combined with the favorable hydrophobic interactions between

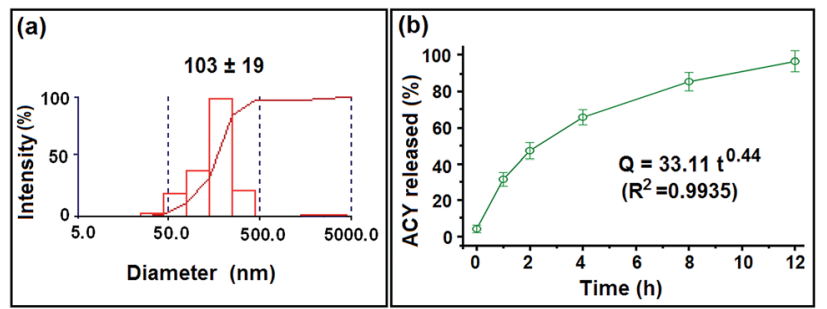

Fig. 3 (a) A typical static and dynamic light scattering analysis $(n=6)$ (b) The in vitro drug release profile $(n=6)$.

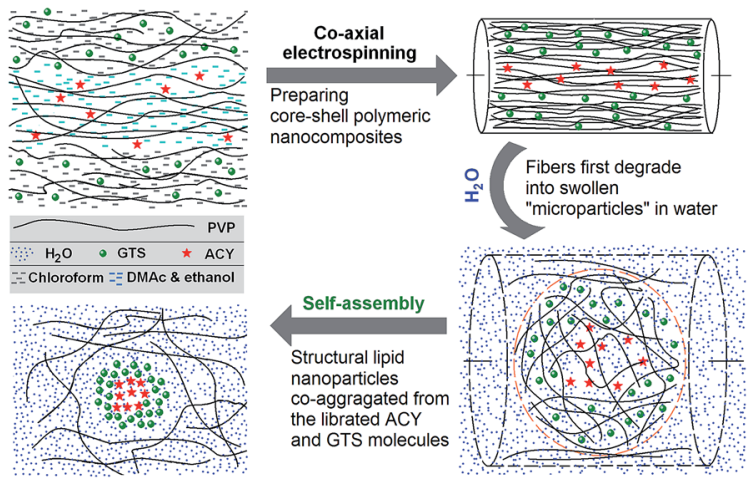

Fig. 4 Process describing the self-assembly of the core-shell nanoparticles from core-shell composite nano-fibers.

the ACY and GTS molecules, allows the transformation of coreshell fibers to core-shell nanoparticles to occur in a controlled, yet spontaneous fashion.

When the fibers were placed into water, the components in them had been already inherently divided into different types according to their solubility in water. The fundamental rules of non-covalent bonding, "Like attracts like", ${ }^{21}$ then can take its role effectively in the confined regions, and based on the fiber structure, can facilitate molecular self-assembly. Moreover, the properties of the fiber mats (big surface area, high porosity and a continuous web structure) and the highly hygroscopic and hydrophilic properties of the matrix polymer PVP favorably ensure the core-shell fibers spontaneously co-assemble into core-shell nanoparticles.

Self-assembly, defined as the autonomous organization of components into ordered patterns or structures, is able to facilitate the creation of a diverse range of hierarchical nanostructures from a wide range of polymeric and non-polymeric materials. ${ }^{22}$ In pharmaceutics, the self-assembly of different types of small molecules in complex supramolecular structures provides a new way in the development of medicated materials for drug delivery applications (particularly for poorly water soluble drugs). ${ }^{23}$ However, the contact of molecules can not be controlled directly on a molecular scale. New methodologies for precisely controlling the assemblies of these molecules as building blocks are important. ${ }^{24,25}$ Pre-positioning the building blocks evenly on a polymer matrix to form a nanocomposite can improve our capability to precisely manipulate molecular transport and contact in a confined nano-scale region. Selfassembly based on core-shell nanocomposites, with the controlled spatial distributions of components, should be easier and more controllable than traditional methods in which agitations at the macro scale are exploited in attempts to control molecular diffusion and to bring the components into contact. ${ }^{26}$ Thus, although here we report on the structural lipid nanoparticles self-assembled from core-shell polymeric composites, many other advanced nano drug delivery systems, such as micelles, liposomes, nanoemulsions, cubosomes, colloidosomes, can be designed and fabricated in a similar way. 
In addition, the core-shell nanostructure is the most fundamental and popular nanostructure, in which the shell can perform a series of functions such as the protection of the core from the outside environment, controlling selective percolation of molecules in and out of the interior of the material and increasing the solubility and biocompatibility of drugs. Many complicated nanostructures are essentially the derivatives of this structure, for example by making holes in the shell or encapsulating even smaller nanoparticles in the core. ${ }^{27}$ It is often thought that the main methods for the generation of coreshell nanoparticles are either bottom-up approaches or topdown approaches, with the former being more suitable. ${ }^{28}$ Presented here is a combined strategy for producing core-shell nanoparticles through transformation at the nanoscale from polymeric core-shell nanocomposites. Electrospinning is developing very quickly in terms of its production scale, ${ }^{29}$ new types of processes, ${ }^{\mathbf{1 3 , 3 0}}$ applications, ${ }^{\mathbf{3 1 , 3 2}}$ and even the new possibility of generating nanofibers from non-polymeric systems. ${ }^{33}$ However, the most fascinating capability of this technology is the generation of core-shell nanofibers. Coaxial electrospinning, ${ }^{\mathbf{1 6}}$ modified coaxial electrospinning, ${ }^{\mathbf{1 1}}$ emulsion electrospinning, ${ }^{34}$ tri-axial electrospinning ${ }^{13}$ and also a combined usage of electrospinning with other techniques such as polymerization ${ }^{35}$ have been reported for generating coreshell nanofibers. These methods should provide new potential templates for manipulating molecular self-assembly.

Finally, polymers have acted as the backbone for the development of novel DDS during the past several decades. Numerous DDS are prepared through a direct encapsulation of drugs in the polymer matrix and depend solely on the physicochemical properties of polymers to achieve a desired release profile or drug pharmacokinetics. ${ }^{36}$ Most recently, polymerlipid combined DDS provided a new potential platform for developing a novel DDS. ${ }^{37,38}$ The present study provides a new example of the combined usage of pharmaceutical polymers and lipid. However, in contrast with previous attempts at this combination, in which the polymers are often water insoluble, ${ }^{37}$ described here is an investigation of the combined application of hydrophilic polymer with lipid. The core-shell nanocomposites are easy to dispense in water due to the hydrophilic polymer matrix, and the subsequently formed lipid nanoparticles are lipophilic that should facilitate the penetration of the drug through the bio-membrane. Thus, the developed coreshell composites should be particularly useful for poorly water soluble drugs of Class IV for both good dispersion/dissolution and cytomembrane penetrability. ${ }^{39}$ Further investigations of these applications are underway.

In summary, a strategy was developed to prepare core-shell lipid nanoparticles through two steps of "copy", i.e. first, to copy the concentric macrostructure of the spinnerets to produce polymeric core-shell nanocomposites through coaxial electrospinning and subsequently produce core-shell lipid nanoparticles through "copy" by molecular self-assembly based on the core-shell nanofibers. The structure of the templates, the components in the core-shell fibers and the surrounding environments acted synergistically to make the self-assembly process accurate and controllable for producing structural lipid nanoparticles in situ with a high encapsulation effect and sustained drug release profiles. This approach can be applied to the creation of self-assembled core-shell nanoparticles from a wide variety of materials systems for different types of applications.

\section{Acknowledgements}

This work was supported by the National Science Foundation of China (nos 51373101 \& 51373100), the China NSFC/UK Royal Society cost share international exchanges scheme (no. 51411130128/IE131748), the Natural Science Foundation of Shanghai (no. 13ZR1428900), the Key Project of the Shanghai Municipal Education Commission (no. 13ZZ113) and the Hujiang Foundation of China (B14006).

\section{Notes and references}

1 J. T. McCann, D. Li and Y. Xia, J. Mater. Chem., 2005, 15, 735. 2 Y. Liu, M. H. Rafailovich, R. Mala, D. Cohn and D. Chidambaram, Proc. Natl. Acad. Sci. U. S. A., 2009, 106, 14201.

3 S. H. Choi, G. Ankonina, D. Y. Youn, S. G. Oh, J. M. Hong, A. Rothschild and I. D. Kim, ACS Nano, 2009, 9, 2623.

4 H. Q. Hou, Z. Jun, A. Reuning, A. Schaper, J. H. Wendorff and A. Greiner, Macromolecules, 2002, 35, 2429.

5 H. Hou and D. Reneker, Adv. Mater., 2004, 16, 69.

6 S. Y. Gu, J. Ren and Q. L. Wu, Synth. Met., 2005, 155, 157.

7 D. G. Yu, L. M. Zhu, S. W. A. Bligh, C. Branford-White and K. White, Chem. Commun., 2011, 47, 1216.

8 D. G. Yu, C. Branford-White, S. W. A. Bligh, G. R. Williams, K. White, L. M. Zhu and N. P. Chatterton, Soft Matter, 2011, 7, 8239.

9 D. G. Yu, G. R. Williams, J. H. Yang, X. Wang, J. M. Yang and X. Y. Li, J. Mater. Chem., 2011, 21, 15957.

10 M. C. George and P. V. Braun, Angew. Chem., Int. Ed., 2009, 48, 8606.

11 D. G. Yu, F. Liu, L. Cui, Z. P. Liu, X. Wang and S. W. A. Bligh, RSC Adv., 2013, 3, 17775.

12 A. Luzio, E. V. Canesi, C. Bertarelli and M. Caironi, Materials, 2014, 7, 906.

13 D. Han and A. Steckl, ACS Appl. Mater. Interfaces, 2013, 5, 8241.

14 J. D. Starr and J. S. Andrew, Chem. Commun., 2013, 49, 4151. 15 Z. P. Liu, L. Cui, D. G. Yu, Z. X. Zhao and L. Chen, Int. J. Nanomed., 2014, 9, 1967.

16 A. K. Moghe and B. S. Gupta, Polym. Rev., 2008, 48(2), 353. 17 D. G. Yu, G. R. Williams, X. Wang, X. K. Liu, H. L. Li and S. W. A. Bligh, $R S C A d v ., 2013,3,4652$.

18 D. Li and Y. Xia, Adv. Mater., 2004, 16, 1151.

19 S. Demirci, A. Celebioglu, Z. Aytac and T. Uyar, Polym. Chem., 2014, 5, 2050.

20 N. A. Peppas, Pharm. Acta Helv., 1985, 60, 110.

21 R. F. Service, Science, 2005, 309, 95.

22 J. J. Panda and V. S. Chauhan, Polym. Chem., 2014, 5, 4418.

23 G. Verma and P. A. Hassan, Phys. Chem. Chem. Phys., 2013, 15, 17016. 
24 C. Hunter, Nature, 2011, 469, 39.

25 G. M. Whiteside and M. Boncheve, Proc. Natl. Acad. Sci. U. S. A., 2002, 99, 4769.

26 H. Hess, Soft Matter, 2006, 2, 669.

27 C. Li, D. G. Yu, G. R. Williams and Z. H. Wang, PLoS One, 2014, 9, 92106.

28 R. G. Chaudhuri and S. Paria, Chem. Rev., 2012, 112, 2373.

29 C. J. Luo, S. D. Stoyanov, E. Stride, E. Pelan and M. Edirisinghe, Chem. Soc. Rev., 2012, 41, 4708.

30 S. L. Liu, Y. Z. Long, Y. Y. Huang, H. D. Zhang, H. W. He, B. Sun, Y. Q. Sui and L. H. Xia, Polym. Chem., 2013, 4, 5696. 31 J. Yan, Y. H. Wu, D. G. Yu, G. R. Williams, S. M. Huang, W. Tao and J. Y. Sun, RSC Adv., 2014, 4, 58265.

32 F. Zheng, S. Wang, M. Shen, M. Zhu and X. Shi, Polym. Chem., 2013, 4, 933.
33 A. Celebioglu and T. Uyar, Nanoscale, 2012, 4, 621.

34 X. Hu, S. Liu, G. Zhou, Y. Huang, Z. Xie and X. Jing, J. Controlled Release, 2014, 185, 12.

35 R. Castagna, R. Momentè, G. Pariani, G. Zerbi, A. Bianco and C. Bertarelli, Polym. Chem., 2014, 5, 6779.

36 K. J. Gandhi, S. V. Deshmane and K. R. Biyani, Int. J. Pharm. Sci. Rev. Res., 2012, 14, 57.

37 K. Hadinoto, A. Sundaresan and W. S. Cheow, Eur. J. Pharm. Biopharm., 2013, 85, 427.

38 L. Zhang, J. M. Chan, F. X. Gu, J. W. Rhee, A. Z. Wang, A. F. Radovic-Moreno, F. Alexis, R. Langer and O. C. Farokhzad, ACS Nano, 2008, 2, 1696.

39 E. Galia, E. Nicolaides, D. Hörter, R. Löbenberg, C. Reppas and J. B. Dressman, Pharm. Res., 1998, 15, 698. 